\title{
Ein Großer aus dem Kreis der chinesischen Medizin ist von uns gegangen
}

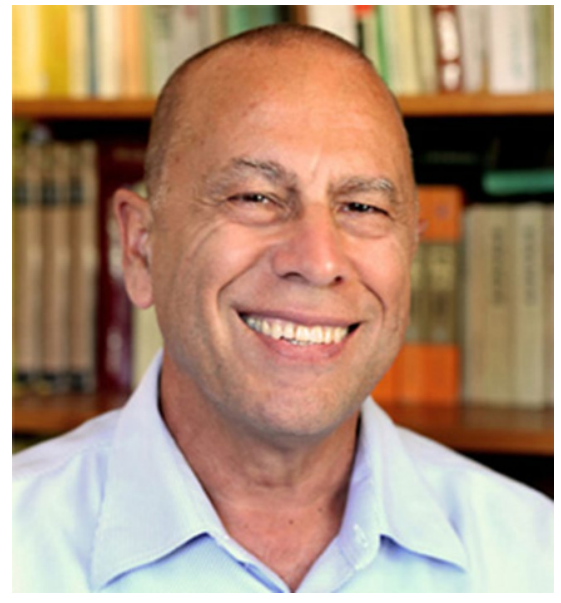

Wollte man sich in den 1980er-Jahren in unseren Breiten umfassend über chinesische Medizin - und nicht nur die „moderne" Version der TCM - informieren, so gab die verfügbare nichtchinesischsprachige Fachliteratur nicht gerade viel her. Doch dann ein Lichtblick, 1989: The Foundations of Chinese Medicine, das Erstlingswerk eines gewissen Giovanni Maciocia aus der Gegend von London, theoretisch fundiert und doch schon auf die klinische Arbeit ausgerichtet. Ich hatte das Glück und die Ehre, dieses und zwei weitere seiner Bücher ins Deutsche übersetzen zu dürfen und ihn bei zahlreichen Kursen im deutschsprachigen Raum zu dolmetschen.

Maciocia war ein unglaublich fleißiger Arbeiter, der anfangs noch neben seiner Praxistätigkeit Werke geradezu enzyklopädischen Ausmaßes verfasste. Diese Haltung spürte man auch in seinen Kursen, wo es ihm um maximale, konzentrierte Wissensvermittlung ging. Da blieb wenig Zeit für Witzeleien, er bestand auf Pünktlichkeit und Ruhe der Teilnehmer, und nach dem Unterricht, den er Punkt für Punkt mit geradezu buchhalterischer Präzision durchzog, waren wir, allen voran Giovanni selbst, stets erschöpft. Aber alle hatten wir viel gelernt. An die 30 Jahre gingen so unzählige Praktizierende der chinesischen $\mathrm{Me}$ dizin durch seine Schule.

Maciocia begnügte sich aber nicht nur mit dem Aufbereiten vorhandenen chinesischen Medizinwissens, sondern entwarf auch auf Basis der Tradition neue Konzep- te, beispielsweise zur wesentlichen Beteiligung der Niere an allergischem und asthmatischem Geschehen. Dieser Ansatz hat sich nicht nur für mich in der täglichen Praxis mannigfach bewährt.

Ihn, der gewohnt war, vor Hunderten Menschen $\mathrm{zu}$ referieren, erfuhr ich im persönlichen Umgang als eher introvertierten Menschen. Er, der geborene Italiener mit Haus an der sonnigen AmalfiKüste, der die letzten Jahre in Kalifornien lebte und arbeitete, ärgerte sich über italienische Kursteilnehmer, die schwätzten und während des Unterrichts mit dem Mobiltelefon hantierten. Er, der so viel Wert auf Präzision und Ordnung legte, äußerte sich immer wieder gegen rigide, konfuzianistische Konzepte in der chinesischen Medizin. Er war eben nicht nur der große Lehrer, sondern vor allem ein Mensch mit all seinen liebenswürdigen immanenten Widersprüchen.

Adieu, Giovanni, und ein großes Dankeschön von der „Generation Maciocia“!

\section{Andreas Höll}

Arzt für Allgemeinmedizin 